\title{
Trombosis asociada a asparaginasa en niños con leucemia linfoblástica aguda
}

\author{
Asparaginase-related thrombosis in children with acute lymphoblastic leukemia
}

\author{
- Carolina Casas', Gloria Ramos², Óscar Eduardo González³, Federico Arroyave ${ }^{4}$ \\ Médica especialista en hematooncología pediátrica. Hospital Infantil Napoleón Franco Pareja (Cartagena). \\ Bacterióloga especialista en hemostasia. Hospital Militar Central. Laboratorio de Referencia en Hemostasia y Hematología H\&H Lab (Bogotá). \\ Médico especialista en hematooncología pediátrica. Fundación Santa Fe. Hospital Central de la Policía Nacional (Bogotá). \\ ${ }^{4}$ Médico especialista en hematooncología pediátrica. Universidad Militar Nueva Granada (Bogotá).
}

\begin{abstract}
Resumen
La asparaginasa es un medicamento fundamental en el tratamiento contra la leucemia linfoblástica aguda. Su uso se asocia a disminución en la síntesis de proteínas que intervienen en el proceso de coagulación y fibrinolisis, lo que conlleva complicaciones trombóticas. La ocurrencia de estos eventos se ha informado después del inicio del tratamiento, reflejando así una posible interacción entre la enfermedad y la terapia.
\end{abstract}

Palabras clave: trombosis, asparaginasa, leucemia linfoblástica aguda, pediatría.

\begin{abstract}
Asparaginase is a key drug in the treatment of acute lymphoblastic leukemia. However, is associated with a decrease in the synthesis of proteins involved in the coagulation and fibrinolysis process, which leads to thrombotic complications. The occurrence of these events has been reported after the initiation of treatment, reflecting a possible interaction between disease and therapy.
\end{abstract}

Key words: Thrombosis, asparaginase, acute lymphoblastic leukemia, pediatric.

\section{Introducción}

La tromboembolia venosa (TEV) es una de las complicaciones más serias en los niños con leucemia linfoblástica aguda (LLA). Su etiología es multifactorial y probablemente está en relación con el aumento en la generación de trombina, sumado al efecto de la quimioterapia. Dentro de los factores de riesgo para trombosis están la presencia de catéter venoso central, las trombofilias congénitas y la terapia antileucémica propiamente dicha. La asparaginasa, crucial en el tratamiento de pacientes con LLA, causa alteraciones hemostáticas que van desde la hemorragia hasta la trombosis, debido a la deficiencia de factores de coagulación y, a su vez, de los anticoagulantes naturales. Para quienes manejan niños con LLA, es difícil establecer qué paciente está en mayor riesgo de trombosis y se beneficie de anticoagulación. Por otro lado, cuando se instaura la trombosis, es un dilema definir la continuidad de la terapia con asparaginasa, teniendo en cuenta la recurrencia del evento y a su vez el impacto en la supervivencia libre de enfermedad. El objetivo de esta revisión es describir el efecto protrombótico de la asparaginasa como factor de riesgo para TEV en niños con LLA, el diagnóstico de estas alteraciones y su manejo.

Con el fin de aclarar este fenómeno, se realizó una búsqueda de la información existente en los últimos 10 años, en PubMed®, incluyendo como palabras clave: acute leukemia, childhood, asparaginase, thrombosis, thromboembolism, coagulation, anticoagulation.

\section{Generalidades}

La asparaginasa es un potente antineoplásico utilizado desde hace aproximadamente 50 años en el manejo de LLA en las fases de inducción a la remisión, consolidación e intensificación. Su introducción en el tratamiento de LLA como agente único permitió

Recibido el 14 de julio de 2017; aceptado el 30 de agosto de 2017 
alcanzar remisiones completas en cerca del $60 \%$ de los pacientes y en combinación con otros agentes en más del 95\%1-5.

Es una enzima derivada de bacterias que convierte la asparagina en ácido aspártico y amoníaco, la cual conduce a una depleción de asparagina, aminoácido indispensable para la viabilidad de las células leucémicas. A diferencia de las células normales, los linfoblastos carecen de la enzima asparagina sintetasa y dependen de los suministros de asparagina exógenos para su supervivencia y proliferación, lo que los hace susceptibles al efecto de la asparaginasa ${ }^{6-7}$.

La inhibición de la síntesis de proteínas es responsable de las principales toxicidades relacionadas con la asparaginasa. La coagulopatía, una complicación conocida de la asparaginasa, se caracteriza por trombosis o hemorragia, siendo más común la primera. Sin embargo, aún se desconocen los factores exactos que inclinan la balanza hacia cualquiera de estas dos condiciones; probablemente se relacione con los medicamentos acompañantes y la secuencia en que se administren?.

Actualmente hay tres preparaciones de asparaginasa disponibles, dos derivadas de Escherichia coli: asparaginasa nativa y asparaginasa pegilada; y una derivada de Erwinia chrysanthemi. Los tres productos comparten el mismo mecanismo de acción, pero tienen diferentes propiedades farmacocinéticas ${ }^{8-10}$.

Aunque es piedra angular en el tratamiento de LLA, se describen complicaciones que pueden amenazar la vida, como hipersensibilidad (10-30\%), pancreatitis (5$10 \%)$, tromboembolia venosa (3.2\%), alteraciones del sistema nervioso central (33\%), hiperglucemia (11-19\%), disfunción hepática (87\%), infecciones (19-29\%), mielosupresión (63-92\%) y segundas neoplasias (1,1-5,4\%) .

La TEV es una condición seria, que ha venido en aumento en los pacientes con LLA, con una tasa de mortalidad general que varía entre el $0 \%$ y el $4,8 \%$, siendo la tromboembolia pulmonar la mayor causa de muerte. La ocurrencia de TEV en esta población de pacientes parece surgir de la interacción de la enfermedad, el tratamiento y la posible predisposición genética para hipercoagulabilidad ${ }^{11}$.

En niños con LLA, la TEV (es decir, trombosis venosa profunda y embolia pulmonar) tiene una incidencia que va del $1,1 \%$ al $36,7 \%$, pero en promedio es del $3,2 \%{ }^{6,11-13}$.
La variabilidad en la incidencia puede explicarse por la diferencia entre los protocolos de tratamiento, la definición del evento (asintomáticos vs. sintomáticos), los métodos para llegar al diagnóstico y el tipo de estudio prospectivo o retrospectivo (Sutor et al., 1999; NowakGottl et al., 2001, 2009; Silverman et al., 2001; Mitchell et al., 2003; Payne \& Vora, 2007) $)^{6,11-13}$.

En LLA, el estado hipercoagulable es de etiología multifactorial y se relaciona probablemente con la gran generación de trombina. Entre los factores que favorecen la trombosis en estos pacientes están las propiedades protrombóticas en sí de las células leucémicas, los factores genéticos, los medicamentos antineoplásicos, como la asparaginasa y los corticoides, la presencia de un catéter venoso central y las complicaciones infecciosas ${ }^{12,13}$.

En pediatría, los pacientes con mayor riesgo de desarrollar TEV son los pacientes mayores de 10 años de edad o aquellos portadores de catéter venoso central, siendo este último el factor de riesgo más frecuente en niños ${ }^{11}$. Racha et al. describen un incremento en la frecuencia de TEV a medida que aumenta la edad: del 2\% (edades 0-5 años), del 20\% (edades 11-14 años) y del $42 \%$ (edad $>30$ años) ${ }^{13}$.

Las trombofilias congénitas, como la mutación del factor $\vee$ Leiden o mutación del gen de la protrombina G20210A, pueden modificar ligeramente el riesgo, pero no son factores de riesgo independientes ${ }^{11}$.

En cuanto al género, no es clara la relación con el desarrollo de TEV. Pui reporta preponderancia en el género masculino, mientras que Nowak-Gottl, Priest y Gugliotta encuentran predominio en mujeres ${ }^{11}$.

Giordano et al. sugieren mayor incidencia de TEV en pacientes con LLA de linaje T de riesgo alto. Los datos del Hospital Infantil McMaster arrojan que el riesgo para TEV es cuatro veces mayor en pacientes con LLA de riesgo alto en comparación con los de riesgo estándar11,14.

Por último, las infecciones graves durante la fase de inducción también aumentan la generación de trombina, con el consiguiente riesgo de trombosis ${ }^{14}$.

\section{Cambios hemostáticos al diagnóstico en niños con LLA}

Hoy en día se ha demostrado a través de modelos experimentales de cáncer en humanos que los oncogenes y algunos genes supresores de tumores son capaces de activar la coagulación. En modelos animales, 
la activación de ciertas vías de señalización por uno o más genes activa la coagulación, la función plaquetaria y suprime la fibrinolisis, como parte de la transformación neoplásica ${ }^{15}$.

Al diagnóstico de la enfermedad, la mayoría de los factores de coagulación e inhibidores se encuentran normales, algunos pueden estar elevados, como el factor VIII, factor de Von Willebrand y fibrinógeno ${ }^{16}$.

Priest, Rodeghiero, Abshire, Mitchell, Oner, Giordano, Uszynky y sus respectivos grupos demostraron un aumento en la generación de trombina al diagnóstico de LLA que se prolonga hasta el primer mes de la terapia, sin que se conozca la etiología exacta de este fenómeno ${ }^{15,16}$.

Los linfoblastos expresan mediadores procoagulantes, fibrinolíticos, proteolíticos y mediadores inflamatorios, como el factor de necrosis tumoral alfa (TNF $\alpha$ ) y la interleuquina 1 beta (IL-1 $\beta$ ), que pueden explicar las alteraciones en la coagulación. Dentro de los agentes procoagulantes están el factor tisular (FT) y el llamado procoagulante del cáncer (PC $)^{15}$. Ambos se sintetizan y se expresan en la superficie de las células tumorales. Sus efectos se ven reforzados por la producción de citoquinas proangiogénicas, como la interleuquina 8 (IL-8) y el factor de crecimiento del endotelio vascular (VEGF), por las células tumorales como por las células endoteliales. Estas citoquinas son capaces de convertir el endotelio anticoagulante en un endotelio procoagulante a través de: 1) la regulación negativa de la expresión de trombomodulina (TM), 2) estimulando la síntesis de FT y del inhibidor del activador del plasminógeno tipo 1 (PAI-1).

Estos hallazgos son concordantes con las investigaciones de Giordano et al., quienes demostraron a través de pruebas de laboratorio que al diagnóstico de la enfermedad hay aumento en la generación de trombina y formación de fibrina (elevación del complejo trombina: antitrombina y dímero D), inhibición de la fibrinólisis (incremento de PAI-1), activación endotelial (niveles altos de multímetros HMW-VWF y P-selectina) e inflamación (aumento de TNF $\alpha$ e IL-6). Después de iniciada la terapia, los marcadores de generación de trombina y de inflamación disminuyeron. Otros, como PAI-1 y P-selectina, aumentaron significativamente, lo que se traduce en perturbación endotelial en relación con la quimioterapia, siendo más evidente durante la fase de inducción con esteroides ${ }^{12,16}$.

\section{Efectos de la terapia con asparaginasa en la hemostasia de pacientes pediátricos con LLA}

A pesar del evidente incremento en la generación de trombina en pacientes con LLA al diagnóstico, la mayoría de los casos de TEV en estos pacientes se reporta solo después del comienzo de la terapia, coincidiendo específicamente con el inicio de la asparaginasa ${ }^{9,13}$. La inhibición de la síntesis proteica por este medicamento es la responsable de la mayoría de las toxicidades, entre ellas la coagulopatía ${ }^{16}$.

Un perfil de coagulación basal anormal en asocio con asparaginasa llevó a la presunción inicial de que su uso por sí solo promovía un estado procoagulante ${ }^{17,18}$. Hoy se sabe que la combinación de asparaginasa con esteroides constituye mayor riesgo 6,11,19

Se postulan cuatro mecanismos que pueden explicar la trombosis relacionada con quimioterapia: 1) la liberación de procoagulantes de acción directa (por ejemplo, TF y PC) y los de acción indirecta (por ejemplo, citoquinas); 2) la toxicidad del fármaco sobre el endotelio vascular; 3) la inducción directa de FT por monocitos y las células tumorales; y 4) la disminución en los anticoagulantes fisiológicos ${ }^{15}$.

El estudio del comportamiento de la hemostasia en pacientes con leucemia es difícil, esto explica los resultados variables entre diferentes investigadores y probablemente se relaciona con el tipo de asparaginasa utilizada, dosis, tiempo en que se toma la muestra (antes o después de la aplicación del medicamento), estatus de la enfermedad, fase del tratamiento, drogas concomitantes y protocolo utilizado ${ }^{16}$. Un enfoque novedoso para reconocer a los pacientes con un mayor riesgo de trombosis asociada a asparaginasa es medir su capacidad de alterar la coagulación in vitro antes del tratamiento 20

En cuanto a la asparaginasa, las principales alteraciones que generan hipercoagulabilidad son:

\section{Disminución de la antitrombina}

La deficiencia de AT relacionada con asparaginasa es la alteración más común dentro de las proteínas de la coagulación. Usualmente se acompaña de otros factores de riesgo trombóticos, como CVC, infecciones, esteroides e inmovilización. Ranta et al. describen el comportamiento de AT en 85 niños con LLA en manejo con asparaginasa, encontrando 
que al diagnóstico los niveles de AT estuvieron normales en todos los niños, pero, una vez iniciado el tratamiento, más del $80 \%$ de ellos tenían niveles de AT bajos. Esta deficiencia fue más evidente luego de la segunda dosis de asparaginasa pegilada. Por otro lado, en presencia de dexametasona (fase de intensificación tardía), la AT aumenta, lo que puede dar lugar a niveles de AT 'satisfactorios'21. La teoría más aceptada que explica la depleción de AT por la asparaginasa es la inhibición de síntesis proteica a nivel hepático. Este hallazgo se hizo evidente al cultivar células HepG2 y someterlas in vitro a asparaginasa, encontrándose un defecto en la producción de la AT, específicamente en el transporte y en la secreción de esta proteína, sin afectarse significativamente la transcripción ${ }^{22}$.

\section{Reducción del fibrinógeno}

Durante la terapia con asparaginasa, el fibrinógeno disminuye de manera dramática. Beinart y Damon describen en adultos que los niveles de fibrinógeno por debajo de 0,5 $\mathrm{g} / \mathrm{l}$ se asociaron con mayor frecuencia de trombosis y proponen a esta proteína como un marcador para identificar un estado de hipercoagulabilidad en adultos con LLA. Consideran entonces que los niveles bajos de fibrinógeno pueden servir como marcador de la reducción de los niveles de proteínas anticoagulantes, tales como la antitrombina, y así predecir el riesgo trombótico. Además, los niveles de fibrinógeno están rápidamente disponibles en comparación con otros niveles de los factores de coagulación, lo que permitiría decisiones clínicas más rápidas ${ }^{23}$. Girolami et al. sugieren que esta tendencia trombótica paradójica en pacientes con fibrinógeno bajo se debe a la persistencia de las grandes cantidades de trombina en circulación que en parte no pueden ser absorbidas por el fibrinógeno circulante ${ }^{24}$.

\section{Deficiencia de proteína C y S}

El tratamiento con asparaginasa también se asocia con la depleción de estos inhibidores naturales ${ }^{16,25}$. La importancia de estas alteraciones en el desarrollo de trombosis aún no es clara. Pui et al. mostraron que no había evidencia directa de que los niveles bajos de estas proteínas influyeran en el desarrollo de TEV en los niños con LLA en la terapia de inducción ${ }^{26}$.

\section{Disminución del plasminógeno}

Además de los fenómenos descritos, también hay disminución de la fibrinolisis, manifiesta por la depleción del plasminógeno. Luego de la primera dosis de asparaginasa, se observa el descenso en los niveles de plasminógeno, acentuándose en presencia de otros medicamentos, como esteroides y citarabina ${ }^{16,25}$.

\section{Aumento de TAFI y PAI-1}

El nivel de TFPI se incrementó notablemente después de la terapia con asparaginasa, lo que indica activación de la vía extrínseca de la coagulación. El deterioro de la capacidad anticoagulante y la disminución de la fibrinolisis debido al plasminógeno bajo y altos niveles de PAI-1 y TAFI son capaces de generar un ambiente favorable para la formación de trombosis ${ }^{25}$.

\section{Incremento de factor tisular y fosfolípidos procoagulantes}

Schneider et al., en su serie de 26 niños con LLA en tratamiento, encontraron que durante terapia con asparaginasa el FT y los fosfolípidos procoagulantes aumentaron con el número de dosis. Este hallazgo es modesto en linfoblastos in vitro. Sin embargo, se observaron niveles plasmáticos más altos de FT después de la octava infusión de asparaginasa ${ }^{27}$.

\section{Manejo}

Los regímenes de tratamiento actuales para LLA en niños y adultos en la mayoría de los casos no proveen una guía para el manejo de TEV asociada a asparaginasa, tampoco el rol de las herramientas imaginológicas, el hecho de continuar o no la asparaginasa ni el método o la duración de la anticoagulación ${ }^{13}$. Mitchell y colaboradores diseñaron un sistema de puntuación para identificar niños con leucemia con mayor riesgo de desarrollar trombosis. El estudio determinó variables que representan un mayor riesgo de trombosis en niños tratados con la estrategia del grupo Berlín-Frankfurt-Münster (BFM): 1) la combinación de prednisona a dosis de $60 \mathrm{mg} / \mathrm{m}^{2} /$ día con asparaginasa, 2) la presencia de un catéter venoso central y 3) la existencia de una trombofilia heredada. Según el estudio, los niños que reciben profilaxis con heparinas de bajo peso molecular (HBPM) clasificados como de 'alto riesgo' 
para trombosis venosa mostraron significativamente menor riesgo de trombosis que los niños sin profilaxis pero con una puntuación de riesgo similar ${ }^{28}$.

\section{Heparinas}

La presencia de niveles adecuados de AT es necesaria para que la heparina tenga efecto anticoagulante. Sin embargo, como se ha descrito anteriormente, en presencia de asparaginasa se espera una depleción de la AT. Por ende, estos pacientes pueden tener anticoagulación inadecuada como resultado de los bajos niveles de AT. Aún no está claro si la AT debe ser monitoreada en todos los pacientes tratados con asparaginasa o solo aquellos con los niveles de anti-Xa subterapéutico ${ }^{13}$.

\section{Hemoderivados}

La terapia de reemplazo con plasma fresco congelado (PFC) o crioprecipitado para hipofibrinogenemia puede aumentar la hipercoagulabilidad y, por ende, el riesgo de trombosis ${ }^{25}$. Las concentraciones de fibrinógeno presentes en el crioprecipitado y de AT en el PFC no pueden ser cuantificadas, por lo tanto, no es posible conocer si los pacientes están siendo tratados de forma adecuada. Adicionalmente, al usar estos productos se expone al paciente a la posibilidad de una reacción transfusional o a la transmisión de un agente infeccioso ${ }^{32}$. En muchos grupos alrededor del mundo se acostumbra a medir de forma rutinaria variables hemostáticas, como el conteo de plaquetas y los niveles de fibrinógeno, y según los resultados se toman decisiones de transfundir plaquetas o crioprecipitado. Esta conducta no está soportada en la literatura ${ }^{33}$ y existe el riesgo teórico de exponer al paciente a mayor riesgo de trombosis. Por otra parte, los concentrados de AT y PFC no eliminan el riesgo de trombosis, lo que sugiere que hay otros componentes en la fisiopatología del TEV ${ }^{21}$. En la tabla 1 se mencionan los principales estudios que existen con respecto al uso de PFC y concentrados de AT en este contexto. Solamente en el estudio de Abbott et al. (2009) se observó menos incidencia de trombosis en sistema nervioso central en los niños que recibieron profilaxis con PFC para deficiencia de ATycrioprecipitadoparahipofibrinogenemia. Todoslosniños que presentaron trombosis tenían LLA de alto riesgo y los autores sugieren que la profilaxis podría estar justificada en este subgrupo de pacientes. Sin embargo, dados los bajos niveles de AT presentes en el PFC y que se ha demostrado en otros estudios que el PFC no tiene impacto significativo sobre las variables hemostáticas, no se justifica el uso de ningún otro producto diferente del concentrado de AT para mantener los niveles normales de esta proteína. Tampoco está clara la frecuencia con que se debe administrar para lograr un impacto significativo ${ }^{33}$.

\section{Suspensión de asparaginasa por trombosis}

La suspensión temprana de la asparaginasa en pacientes pediátricos por eventos trombóticos $u$ otras toxicidades lamentablemente se asocia a una supervivencia libre de enfermedad más baja². Sin embargo, esta medida sigue siendo recomendada por algunos autores cuando la trombosis es clínicamente importante $^{31}$. La reexposición con asparaginasa es factible y segura bajo el manejo con heparina profiláctica; así lo demostraron Qureshi et al.29. Grace et al. agregan

Tabla 1. Comparación de estudios donde se evaluó la profilaxis con PFC, crioprecipitado o concentrado de AT en pacientes con LLA en terapia con asparaginasa

\begin{tabular}{|c|c|c|c|}
\hline Referencia & Intervención & Tamaño de muestra & Resultado \\
\hline Nowak-Gottl et al. (1995) & $\begin{array}{l}\text { Infusión de PFC de donante único cuando había } \\
\text { niveles de fibrinógeno por debajo de } 0,6 \mathrm{~g} / \mathrm{l} \text {. }\end{array}$ & 20 niños (42 dosis) & $\begin{array}{l}\text { - No mejoría clínica. } \\
\text { - Mínimo aumento de fibrinógeno y de } \\
\text { niveles de alfa-1 antiplasmina. } \\
\text { - No cambios en AT y en plasminógeno. }\end{array}$ \\
\hline Halton et al. (1994) & PFC 20 cc/kg luego de infusión de asparaginasa. & 8 niños & $\begin{array}{l}\text { No hubo cambios significativos, clínicos ni } \\
\text { estadísticos en las variables hemostáticas. }\end{array}$ \\
\hline Gugliotta et al. (1992) & $\begin{array}{l}\text { Suplementación de AT } 2.000 \text { unidades intravenosas } \\
\text { en días alternos después de la segunda infusión de } \\
\text { asparaginasa. }\end{array}$ & 15 adultos & $\begin{array}{l}\text { Normalización de los niveles de dímero } \\
\text { D y de los niveles de complejo trombina- } \\
\text { antitrombina. }\end{array}$ \\
\hline Mitchell et al. (2003). Estudio Parkaa & $\begin{array}{l}\text { Estudio aleatorizado, suplementación de } \\
\text { concentrado de AT en días } 1,8,15,22 \text { versus no } \\
\text { suplementación. }\end{array}$ & $\begin{array}{l}109 \text { niños ( } 25 \text { recibieron AT, } 60 \text { no recibieron } \\
\text { AT) }\end{array}$ & $\begin{array}{l}\text { - Trombosis }=7 / 25 \text { ( } 28 \%) \text { en brazo de AT, } \\
22 / 60 \text { en brazo de no AT. } \\
\text { - P no significativa. } \\
\text { - Mayoría asintomáticos. }\end{array}$ \\
\hline Abbott et al. (2009) & $\begin{array}{l}\text { Dos centros: IWK suplementó con PFC si AT menor } \\
\text { de } 50 \mathrm{u} / \mathrm{ml} \text { y crioprecipitado si fibrinógeno menor } \\
\text { de } 1 \mathrm{~g} / \mathrm{l} \text {. En BCCH no daban ninguna profilaxis. }\end{array}$ & $\begin{array}{l}\text { IKW } 240 \\
\text { BCCH } 479\end{array}$ & $\begin{array}{l}\text { - En IWK: cero trombosis de sistema nervioso } \\
\text { central. } \\
\text { - En BCCH: } 7 \text { trombosis de sistema nervioso } \\
\text { central (1,5\%). } \\
\text { - Sugiere que la profilaxis no está justificada } \\
\text { en forma generalizada, quizá solo en } \\
\text { pacientes con LLA de riesgo alto. }\end{array}$ \\
\hline
\end{tabular}

PFC: plasma fresco congelado. AT: antitrombina. IWK: Hospital de niños Izaak Walton Killam. BCCH: Hospital de niños de British Columbia. LLA: leucemia linfoblástica aguda. g/l: gramos por litro. u/ml: unidades por mililitro.

Fuente: tomado de Astwood et al. (2011)33. 
que la recurrencia de eventos trombóticos durante el tratamiento con asparaginasa pudiera ser minimizada siguiendo los niveles de anti-Xa y AT durante la terapia con heparina ${ }^{30}$.

\section{Conclusiones y consideraciones futuras}

La trombosis venosa (TEV) es una condición seria que ha venido en aumento en los pacientes con LLA. La asparaginasa es parte fundamental del tratamiento de inducción de LLA, se asocia a disminución de la síntesis proteica de los factores de la coagulación y del sistema fibrinolítico, pero por sí sola no parece ser el mayor determinante de trombosis. Este fenómeno se debe muy probablemente a la interacción de la enfermedad, el tratamiento y la predisposición genética para hipercoagulabilidad de cada individuo. Una vez establecida la trombosis, algunos expertos recomiendan la suspensión temporal de este medicamento, lo que afecta negativamente la supervivencia. En niños, los esfuerzos para minimizar el riesgo de tromboembolia aún son limitados. El sistema desarrollado por Mitchel et al. es el primer paso para la categorización de riesgo y para determinar quiénes se benefician de la anticoagulación profiláctica. La anticoagulación en niños con leucemia es un reto y aún son necesarios más estudios donde se tengan en cuenta el riesgo genético y el relacionado con el tratamiento.

\section{Referencias}

1. Silverman $L B$, Declerck $L$, Gelber RD, Dalton VK, Asselin $B L$, Barr $\mathrm{RD}$, et al. Results of Dana-Farber Cancer Institute Consortium protocols for children with newly diagnosed acute lymphoblastic leukemia (1981-1995). Leukemia. 2000;14(12):2247-56.

2. Silverman LB, Gelber RD, Dalton VK, Asselin BL, Barr RD, Clavell LA, et al. Improved outcome for children with acute lymphoblastic leukemia: results of Dana-Farber Consortium Protocol 91-01. Blood. 2001;97(5):1211-8.

3. Pui $\mathrm{CH}$, Sandlund JT, Pei $D$, Campana $D$, Rivera GK, Ribeiro $R C$, et al. Improved outcome for children with acute lymphoblastic leukemia: results of Total Therapy Study XIIIB at St Jude Children's Research Hospital. Blood. 2004;104(9):2690-6.

4. Pui $C H$, Campana D, Pei D, Bowman WP, Sandlund JT, Kaste SC, et al. Treating childhood acute lymphoblastic leukemia without cranial irradiation. N Engl J Med. 2009;360(26):2730-41.

5. Möricke A, Reiter A, Zimmermann $M$, Gadner $H$, Stanulla $M$, Dördelmann $M$, et al. Risk-adjusted therapy of acute lymphoblastic leukemia can decrease treatment burden and improve survival: treatment results of 2169 unselected pediatric and adolescent patients enrolled in the trial ALL-BFM 95. Blood. 2008;111(9):4477-89
6. Raetz EA, Salzer WL. Tolerability and efficacy of L-asparaginase therapy in pediatric patients with acute lymphoblastic leukemia. J Pediatr Hematol Oncol. 2010;32(7):554-63.

7. Athale UH, Chan AK. Thrombosis in children with acute lymphoblastic leukemia: Part II. Pathogenesis of thrombosis in children with acute lymphoblastic leukemia: effects of the disease and therapy. Thromb Res. 2003;111(4-5):199-212.

8. Pieters $R$, Hunger SP, Boos J, Rizzari C, Silverman L, Baruchel A, et al. L-asparaginase treatment in acute lymphoblastic leukemia: a focus on Erwinia asparaginase. Cancer. 2011;117(2):238-49.

9. Rizzari C, Conter V, Starý J, Colombini A, Moericke A, Schrappe M. Optimizing asparaginase therapy for acute lymphoblastic leukemia. Curr Opin Oncol. 2013;25 Suppl 1:S1-9.

10. Andrade AF, Borges KS, Silveira VS. Update on the use of Lasparaginase in infants and adolescent patients with acute lymphoblastic leukemia. Clin Med Insights Oncol. 2014;8:95-100.

11. Athale UH, Chan AK. Thrombosis in children with acute lymphoblastic leukemia: part I. Epidemiology of thrombosis in children with acute lymphoblastic leukemia. Thromb Res. 2003;111(3):125-31.

12. Giordano P, Molinari AC, Del Vecchio GC, Saracco P, Russo G, Altomare $\mathrm{M}$, et al. Prospective study of hemostatic alterations in children with acute lymphoblastic leukemia. Am J Hematol. 2010;85(5):325-30.

13. Grace RF, Dahlberg SE, Neuberg D, Sallan SE, Connors JM, Neufeld EJ, et al. The frequency and management of asparaginaserelated thrombosis in paediatric and adult patients with acute lymphoblastic leukaemia treated on Dana-Farber Cancer Institute consortium protocols. Br J Haematol. 2011;152(4):452-9.

14. Lejhancova-Tousovska K, Zapletal O, Vytiskova S, Strbackova P, Sterba J. Profile of thrombin generation in children with acute lymphoblastic leukemia treated by Berlin-Frankfurt-Münster (BFM) protocols. Blood Coagul Fibrinolysis. 2012;23(2):144-54

15. Rickles FR, Falanga A, Montesinos P, Sanz MA, Brenner B, Barbui T. Bleeding and thrombosis in acute leukemia: what does the future of therapy look like? Thromb Res. 2007;120 Suppl 2:599-106

16. Athale UH, Chan AK. Thrombosis in children with acute lymphoblastic leukemia. Part II. Pathogenesis of thrombosis in children with acute lymphoblastic leukemia: effects of the disease and therapy. Thromb Res. 2003;111(4-5):199-212.

17. Priest JR, Ramsay NK, Steinherz PG, Tubergen DG, Cairo MS, Sitarz AL, et al. A syndrome of thrombosis and hemorrhage complicating L-asparaginase therapy for childhood acute lymphoblastic leukemia. J Pediatr. 1982;100(6):984-9.

18. Shapiro AD, Clarke SL, Christian JM, Odom LF, Hathaway WE. Thrombosis in children receiving L-asparaginase. Determining patients at risk. Am J Pediatr Hematol Oncol. 1993;15(4):400-5.

19. Nowak-Göttl U, Heinecke $A$, von Kries $R$, Nürnberger W, Münchow N, Junker R. Thrombotic events revisited in children with acute lymphoblastic leukemia: impact of concomitant Escherichia coli asparaginase/prednisone administration. Thromb Res. 2001;103(3):165-72.

20. Stief TW, Chalkitis D. Asparaginase modulates thrombin generation. Hemostasis Lab. 2008;1:303-66.

21. Ranta S, Heyman MM, Jahnukainen $K$, Taskinen M, SaarinenPihkala UM, Frisk T, et al. Antithrombin deficiency after prolonged asparaginase treatment in children with acute lymphoblastic leukemia. Blood Coagul Fibrinolysis. 2013;24(7):749-56. 
22. Bushman JE, Palmieri D, Whinna HC, Church FC. Insight into the mechanism of asparaginase-induced depletion of antithrombin III in treatment of childhood acute lymphoblastic leukemia. Leuk Res. 2000;24(7):559-65.

23. Beinart G, Damon L. Thrombosis associated with L-asparaginase therapy and low fibrinogen levels in adult acute lymphoblastic leukemia. Am J Hematol. 2004;77(4):331-5.

24. Girolami A, Ruzzon E, Tezza F, Scandellari R, Vettore S, Girolami B. Arterial and venous thrombosis in rare congenital bleeding disorders: a critical review. Haemophilia. 2006;12(4):345-51.

25. Albayrak M, Gürsel T, Kaya Z, Koçak U. Alterations in procoagulant, anticoagulant, and fibrinolytic systems before and after start of induction chemotherapy in children with acute lymphoblastic leukemia. Clin Appl Thromb Hemost. 2013;19(6):644-51.

26. Pui $\mathrm{CH}$, Chesney CM, Bergum PW, Jackson CW, Rapaport SI. Lack of pathogenetic role of proteins $C$ and $S$ in thrombosis associated with asparaginase-prednisone-vincristine therapy for leukaemia. Br J Haematol. 1986;64(2):283-90.

27. Schneider $P$, Van Dreden $P$, Rousseau $A$, Kassim $Y$, Legrand $E$, Vannier JP, et al. Increased levels of tissue factor activity and procoagulant phospholipids during treatment of children with acute lymphoblastic leukaemia. $\mathrm{Br} J$ Haematol. 2010;148(4):582-92.
28. Mitchell L, Lambers $M$, Flege $S$, Kenet $G$, Li-Thiao-Te $V$, Holzhauer $S$, et al. Validation of a predictive model for identifying an increased risk for thromboembolism in children with acute lymphoblastic leukemia: results of a multicenter cohort study. Blood. 2010;115(24):4999-5004.

29. Qureshi A, Mitchell C, Richards S, Vora A, Goulden N. Asparaginase-related venous thrombosis in UKALL 2003- reexposure to asparaginase is feasible and safe. $\mathrm{Br} J$ Haematol. 2010;149(3):410-3

30. Grace RF, Dahlberg SE, Neuberg D, Sallan SE, Connors JM, Neufeld EJ, et al. The frequency and management of asparaginaserelated thrombosis in paediatric and adult patients with acute lymphoblastic leukaemia treated on Dana-Farber Cancer Institute consortium protocols. Br J Haematol. 2011;152(4):452-9.

31. Hijiya N, Van der Sluis IM. Asparaginase-associated toxicity in children with acute lymphoblastic leukemia. Leuk Lymphoma. 2016;57(4):748-57.

32. Franchini M, Lippi G. Fibrinogen replacement therapy: a critical review of the literature. Blood Transfus. 2012;10(1):23-7.

33. Astwood E, Vora A. Personal practice: how we manage the risk of bleeding and thrombosis in children and young adults with acute lymphoblastic leukemia. $\mathrm{Br} J$ Haematol 2011;152(5):505-11. 